\title{
Impacto del deterioro cognitivo sobre la salud oral en personas mayores
}

\author{
Ana Adamuz Jiménez, Antonio García Rubio y \\ María Cristina Ibáñez Romero \\ Servicio Andaluz de Salud (España)
}

\begin{abstract}
El deterioro cognitivo y la demencia son trastornos que se caracterizan por una disminución de la cognición que afecta a uno o más aspectos de la misma, llegando a interferir con las actividades de la vida diaria de la persona. Estudios epidemiológicos han mostrado claras evidencias que relacionan el estado de salud oral con el deterioro cognitivo/demencias. El objetivo del presente trabajo es conocer el impacto del deterioro cognitivo en la salud oral de una población mayor. Se ha realizado un estudio secundario a partir de los datos del estudio Periodontitis-Demencia, estudio de casos y controles realizado en Granada con 564 pacientes mayores de 65 años. Los casos fueron mayores diagnosticados de deterioro cognitivo o demencias y los controles sujetos sanos sin deterioro cognitivo. Se recogieron datos sobre higiene oral, número de dientes presentes, CAOD, índice de placa, índice de sangrado, profundidad de bolsa al sondaje y pérdida de inserción epitelial entre otros. Se ha observado una asociación estadísticamente significativa entre nivel de deterioro cognitivo y estado de salud oral, encontrando un mayor nivel de índice de placa y sangrado. Así como una relación entre la extensión de enfermedad periodontal y gravedad de deterioro cognitivo, una vez ajustado por las variables que pueden afectar a esta asociación como son la edad, sexo, nivel de estudios, tabaco o alcohol. Existe una relación estadísticamente significativa entre el nivel de deterioro cognitivo y el estado de salud periodontal.
\end{abstract}

Palabras clave: Deterioro cognitivo leve, demencia, salud oral, periodontitis.

Impact of cognitive impairment on oral health in the elderly. Cognitive impairment and dementia are disorders characterized by decreased cognition that affects one or more aspects of it, coming to interfere with activities of daily living of the person. Epidemiological studies have shown strong evidence of association between oral health status and cognitive impairment/dementia. The aim of this study was to determinate the impact of cognitive impairment on oral health of a eldery population. It has been conducted a study based on secondary data of Periodontitis-Dementia study, a case-control study conducted in Granada with 564 patients over 65 years. Cases were older people diagnosed with cognitive impairment or dementia and controls were healthy subjects without cognitive impairment. Data collected were: oral hygiene, teeth present, DMFT, plaque index, bleeding index, probing depth and clinical attachment loss. A statistically significant association was observed between level of cognitive impairment and oral health status, finding a higher level of plaque and bleeding indexes and an association between the extent of periodontal disease and severity of cognitive impairment, after controlling for confounding variables such age, sex, educational level, tobacco or alcohol. There is a statistically significant association between level of cognitive impairment and periodontal health status.

Keywords: Mild cognitive impairment, dementia, oral health, periodontitis.

Correspondencia: Ana Adamuz Jiménez. Avd. Luis Miranda Davalos, n⿳9, portal $1,3^{\circ} \mathrm{C}$. C.P.: 18014. Granada (España). E-mail: ana273ad@ hotmail.com 
El deterioro cognitivo y la demencia son trastornos que se caracterizan por una disminución de la cognición que afecta a uno o más aspectos de la misma, como son el aprendizaje y la memoria, el lenguaje, las funciones ejecutivas, la atención compleja, funciones perceptivo-motrices o la cognición social (Brennan y Strauss, 2014).

Se considera deterioro cognitivo al estado intermedio que aparece con frecuencia antes del desarrollo de la demencia, en el que el deterioro cognitivo y conductual no es suficientemente grave como para tener repercusiones funcionales (Petersen, 2011). Cuando estos déficits en la cognición interfieren con la autonomía e independencia en las actividades básicas de la vida diaria de la persona (como por ejemplo el aseo personal), hablamos de demencia, siempre y cuando las alteraciones producidas no se expliquen mejor por otro trastorno mental (Brennan et al., 2014).

A pesar de que la demencia es una enfermedad multietiológica, la enfermedad de Alzheimer es el tipo más común de presentación, representando un 60-70\% de todos los casos (Noble, Scarmeas, y Papapanou, 2013). La edad sigue siendo el factor de riesgo más importante en el desarrollo de la demencia, en particular para la enfermedad de Alzheimer. La incidencia de esta enfermedad aumenta exponencialmente a partir de los 65 años (15 casos/1.000 personas), llegando a 69.2 casos/1.000 personas-año en las personas con edades superiores a los 90 años en nuestro medio (Bermejo-Pareja, BenitoLeón, Vega, Medrano, y Román, 2008). En España se estima una prevalencia de 600.000 pacientes con demencias y 400.000 de enfermedad de Alzheimer (Pedro-Cuesta et al., 2009).

Existen claras evidencias científicas de una relación entre el estado de salud oral y las demencias o deterioro cognitivo. Esta asociación, tiene, sin embargo, una doble dirección. Por un lado, parece lógico pensar que, en los mayores con una clara progresión en su deterioro cognitivo y pérdida de funcionalidad e independencia, disminuya su capacidad para mantener la higiene oral y acudir a los servicios de odontología, público y/o privados. Esto los lleva irremediablemente a presentar estados de salud oral lejos de lo deseable (Lee, Wu, y Plassman, 2013; Martande et al., 2014; Naorungroj et al., 2013). Pero, además, en los últimos años se ha presentado la salud oral como un posible factor de riesgo para la instauración de una demencia o deterioro cognitivo, basándose en la hipótesis de la infección/inflamación de origen dental como detonante del daño cerebral (Gil-Montoya et al., 2015; Kamer et al., 2008; Singhrao et al., 2014).

En esta ocasión, exploraremos la primera vía de relación comentada, es decir, la demencia como causante del estado de salud oral de los mayores. El objetivo del presente trabajo es conocer el impacto del deterioro cognitivo en la salud oral de una población mayor. 


\section{MÉTODO}

\section{Diseño y población de estudio}

Se trata de un estudio que deriva de los datos recogidos en un proyecto más amplio denominado Periodontitis-Demencia llevado a cabo por la Universidad de Granada. En dicho proyecto original, se estudia la periodontitis como factor de riesgo frente a la demencia. Para ello, se diseñó un estudio de casos/control con una estimación de tamaño muestral mínimo de 182 sujetos por grupo. Para un error alpha del 5\%, y potencia del $80 \%$, esos tamaños muestrales permitían detectar una diferencia del $15 \%$ (diferencia clínica pequeña) en la prevalencia de EP entre casos y controles, considerando a priori la más desfavorable de las situaciones $(p=q=0.50)$. Para la consecución de este estudio secundario, hemos seleccionado y analizado el total de la muestra, tanto dentados como desdentados.

Los casos, fueron reclutados entre los meses de enero de 2011 y julio de 2012 en los Servicios de Neurología de los Hospitales Universitarios Virgen de las Nieves de Granada y San Cecilio. Los controles fueron sujetos que demandaban asistencia médica no odontológica en el mismo periodo y en un centro de salud cercano al hospital de referencia y de 4 centros geriátricos de la ciudad de Granada. Todos los pacientes firmaron el consentimiento informado. El Comité Ético de la Universidad de Granada valoró positivamente el proyecto.

\section{Participantes}

La selección de los casos y controles se realizó tratando de homogeneizar los grupos en cuanto a edad, sexo y nivel de estudios, procediendo los pacientes de la misma unidad geográfica. Para la determinación de un "caso", pacientes con deterioro cognitivo con o sin demencia de cualquier etiología y gravedad, se han seguido los protocolos previamente establecidos por los Servicios de Neurología participantes en el estudio, centrados en una anamnesis orientada, una exploración general y neurológica, escalas psicométricas adaptadas a la población española y pruebas complementarias. Los "controles" fueron sujetos que demandaban tratamiento médico en un centro de salud y sujetos que residían en centros geriátricos. Se seleccionaron aquellos que no presentaban diagnóstico ni sospecha de cualquier tipo de demencia o pérdida episódica de memoria. En los casos sospechosos se realizó un test de screening denominado Test de las Fotos (Carnero-Pardo y Montoro-Ríos, 2004).

Los criterios de inclusión generales en ambos grupos fueron tener más de 65 años, con o sin dientes. En esta ocasión se han incluido sujetos desdentados por entender la variable "pérdida de dientes" como una variable proxy relacionado con la enfermedad periodontal. Se excluyeron sujetos con graves alteraciones del sistema inmunitario y 
sujetos con deterioro cognitivo grave que le impidiera una mínima colaboración en la exploración oral.

\section{Valoración de la salud oral}

De manera genérica se siguieron las recomendaciones de la OMS para la recogida de datos sobre salud oral y los criterios publicados por Beck y Löe para la exploración periodontal (Beck y Löe, 1993). La exploración bucodental la realizaron dos encuestadores previamente formados y calibrados (diferentes a la autora de esta memoria) para homogeneizar los criterios de evaluación periodontal básicamente. Para la recogida de datos periodontales se trató de explorar el máximo número de dientes presentes, siempre que lo permitiera el estado físico del paciente. La misma exploración oral y periodontal se realizó en el grupo de casos como en los controles.

En la historia clínica dental se registraron datos sobre la higiene oral del paciente, el número de dientes presentes, CAOD, índice de placa bacteriana, el índice de sangrado expresado en porcentaje, la profundidad de bolsa al sondaje y la pérdida de inserción epitelial (porcentaje de sitios con pérdida de inserción epitelial $\geq$ a $3 \mathrm{~mm}$ ).

Se recogieron también variables sociodemográficas y otras como los antecedentes familiares de deterioro cognitivo o demencia, antecedentes personales de hábitos tóxicos, factores de riesgo cerebrovasculares, enfermedades endocrinometabólicas, infecciones, alteraciones respiratorias, enfermedades psiquiátricas (datos no reflejados en esta memoria). Los antecedentes farmacológicos se basaron en los fármacos que pudieran afectar negativamente el rendimiento cognitivo (antidepresivos, benzodiacepinas, anticonvulsivantes, neurolépticos, etc.).

\section{Análisis estadístico}

Se ha llevado a cabo un análisis descriptivo, bivariante y multivariante para determinar si existen asociaciones entre las diferentes variables de salud oral y el deterioro cognitivo. Se han utilizado el test de Chi-Cuadrado, Test de Mann-Whitney y Test de Student para la comparación de variables categóricas y comparación de medias. Los sujetos desdentados se excluyeron para el análisis de las variables periodontales para poder conocer realmente la influencia del estado periodontal en la variable resultado y no depender de la variable "proxy". Hemos utilizado el análisis regresión logística para modelar la asociación entre el estado de salud oral y el deterioro cognitivo. Para ello se han construido varios modelos de regresión, tomando como variables dependientes el número de dientes presentes, CAOD, índice de placa y sangrado, profundidad de sondaje y pérdida de inserción epitelial y como variables independientes en todos los modelos el deterioro cognitivo categorizado en SI/NO. Los modelos se ajustaron por edad, sexo, nivel de estudios y consumo de tabaco y alcohol. Los datos han sido analizados con el paquete estadístico SPSS 17.0. 


\section{RESULTADOS}

Se obtuvo una muestra total de 564 sujetos, de los cuales 324 eran controles, 107 pacientes con deterioro cognitivo leve o demencia y 133 con demencia moderada o severa.

La edad media de la muestra fue de 80 y 79.8 años para mayores con deterioro cognitivo moderado/severo y controles respectivamente. En todos los grupos fue mayor el porcentaje de mujeres sobre hombres, siendo esta diferencia más destacada en el grupo de demencia moderada y severa (Tabla 1).

Tabla 1. Asociación bivariante entre deterioro cognitivo (casos) y variables estudiadas $(n=564)$

\begin{tabular}{|c|c|c|c|c|c|}
\hline Variable & $\begin{array}{l}\text { Controles } \\
(n=324)[\mathrm{A}]\end{array}$ & $\begin{array}{l}\mathrm{DCL}^{1 /} \text { Demencia } \\
\text { leve [B] }(n=107)\end{array}$ & $\begin{array}{l}\text { Demecia moderada y } \\
\text { severa }[C](n=133)\end{array}$ & $\begin{array}{l}\text { Comparación } \\
\text { global } p \text {-valor }\end{array}$ & $\begin{array}{l}\text { Comparaci } \\
\text { ón por } \\
\text { pares }^{\mathrm{g}}\end{array}$ \\
\hline $\begin{array}{c}\text { Edad (años), } \\
\text { media } \pm \text { sd }\end{array}$ & $79.8 \pm 8.3$ & $76.1 \pm 7.4$ & $80.0 \pm 7.5$ & $<0.001^{\mathrm{d}}$ & $\mathrm{B} \neq \mathrm{A}, \mathrm{C}^{\mathrm{h}}$ \\
\hline \multicolumn{6}{|l|}{ Edad (años), n (\%) } \\
\hline $51-69$ & $(12.3)$ & $(12.1)$ & $(9.0)$ & & \\
\hline $70-79$ & $112 \quad(34.6)$ & $(58.9)$ & $(35.3)$ & & \\
\hline $80-89$ & $(40.1)$ & $(27.1)$ & $(45.9)$ & & \\
\hline $90-98$ & $(13.0)$ & (1.9) & $(9.8)$ & & \\
\hline \multicolumn{6}{|l|}{ Sexo, $n(\%)$} \\
\hline Hombre & $\begin{array}{ll}135 & (41.7) \\
\end{array}$ & $(40.2)$ & $(29.3)$ & \multirow[t]{2}{*}{$0.044^{\mathrm{e}}$} & \multirow[t]{2}{*}{$\mathrm{A} \neq \mathrm{C}^{\mathrm{i}}$} \\
\hline Mujer & $189 \quad(58.3)$ & $(59.8)$ & $(70.7)$ & & \\
\hline \multicolumn{6}{|l|}{ Estudios, $n(\%)$} \\
\hline Univ/Secundaria & $(12.0)$ & $(7.5)$ & $(8.3)$ & \multirow{5}{*}{$0.193^{\mathrm{f}}$} & \\
\hline Primaria & $(19.1)$ & $(25.2)$ & (18.0) & & \\
\hline Primaria incompl. ${ }^{\mathrm{a}}$ & $127 \quad(39.2)$ & $(49.5)$ & $(42.9)$ & & \\
\hline Ninguno & $(29.6)$ & $(17.8)$ & $(30.8)$ & & \\
\hline Univ/Secundaria & $(12.0)$ & $(7.5)$ & $(8.3)$ & & \\
\hline \multicolumn{4}{|l|}{ Tabaco, $n(\%)$} & \multirow{3}{*}{$0.030^{\mathrm{e}}$} & \multirow{3}{*}{$\mathrm{A} \neq \mathrm{C}^{\mathrm{i}}$} \\
\hline Sí & $\begin{array}{ll}33 & (10.2) \\
\end{array}$ & $(6.5)$ & $(3.0)$ & & \\
\hline $\mathrm{No}^{\mathrm{b}}$ & $(89.8)$ & $(93.5)$ & $(97.0)$ & & \\
\hline \multicolumn{4}{|l|}{ Alcohol, $n(\%)$} & \multirow{4}{*}{$0.020^{\mathrm{f}}$} & \multirow{4}{*}{$\mathrm{B} \neq \mathrm{A}, \mathrm{C}^{\mathrm{j}}$} \\
\hline$\geq 1$ vez/día & $13 \quad(4.0)$ & $(8.4)$ & $(2.3)$ & & \\
\hline Eventualmente & $(10.8)$ & $(15.9)$ & $(9.8)$ & & \\
\hline $\mathrm{No}^{\mathrm{c}}$ & $276 \quad(85.2)$ & $(75.7)$ & $(88.0)$ & & \\
\hline \multicolumn{4}{|l|}{ Cepill. dental, $n(\%)$} & \multirow{5}{*}{$<0.001^{\mathrm{f}}$} & \multirow{5}{*}{$\mathrm{A} \neq \mathrm{B} \neq \mathrm{C}^{\mathrm{j}}$} \\
\hline No & $(9.3)$ & $(5.6)$ & $(33.8)$ & & \\
\hline $\begin{array}{c}\text { Solo enjuague } \\
\text { bucal }\end{array}$ & (7.7) & $(17.8)$ & $(26.3)$ & & \\
\hline 1 vez/día & $118 \quad(36.4)$ & $(44.9)$ & (28.6) & & \\
\hline$\geq 2$ veces/día & $\begin{array}{ll}151 & (46.6) \\
\end{array}$ & $(31.8)$ & $(11.3)$ & & \\
\hline
\end{tabular}

Pese a que existen algunas diferencias entre el grupo control y los casos destaca principalmente la higiene oral. En este caso se observa una diferencia significativa entre el número de veces que realizan el cepillado dental, existiendo además un claro descenso a medida que aumenta el grado de deterioro cognitivo. De tal manera 
que un $46.6 \%$ del grupo control lo realiza dos veces al día, mientras que esta cifra disminuye hasta un $31.8 \%$ y un $11.3 \%$ en los grupos de deterioro cognitivo leve y de demencia moderada-severa respectivamente. Así mismo, un 33.8\% del grupo demencia moderada y severa no se cepilla nunca, frente al $9.3 \%$ que no lo hace del grupo control. El control de la placa bacteriana en esta población es quizás la clave y explicación del estado de salud oral evidenciado.

En el análisis bivariado entre deterioro cognitivo y variables dentales (Tabla 2) se observa que no existen diferencias significativas en el número de dientes presentes entre un grupo y otro. En el grupo control el $29.3 \%$ de los pacientes eran desdentados y el $24.7 \%$ en los pacientes con deterioro cognitivo-demencia. En algunos trabajos previos sí que existe dicha asociación, presentando la variable pérdida de dientes claramente asociada al diagnóstico de deterioro cognitivo o a la demencia (Lexomboon, Trulsso, Wårdh, y Parker, 2012; Nilsson, Berglund, y Renvert, 2014).

Tabla 2. Asociación bivariante entre deterioro cognitivo (casos) y variables dentales ( $n=564 a)$

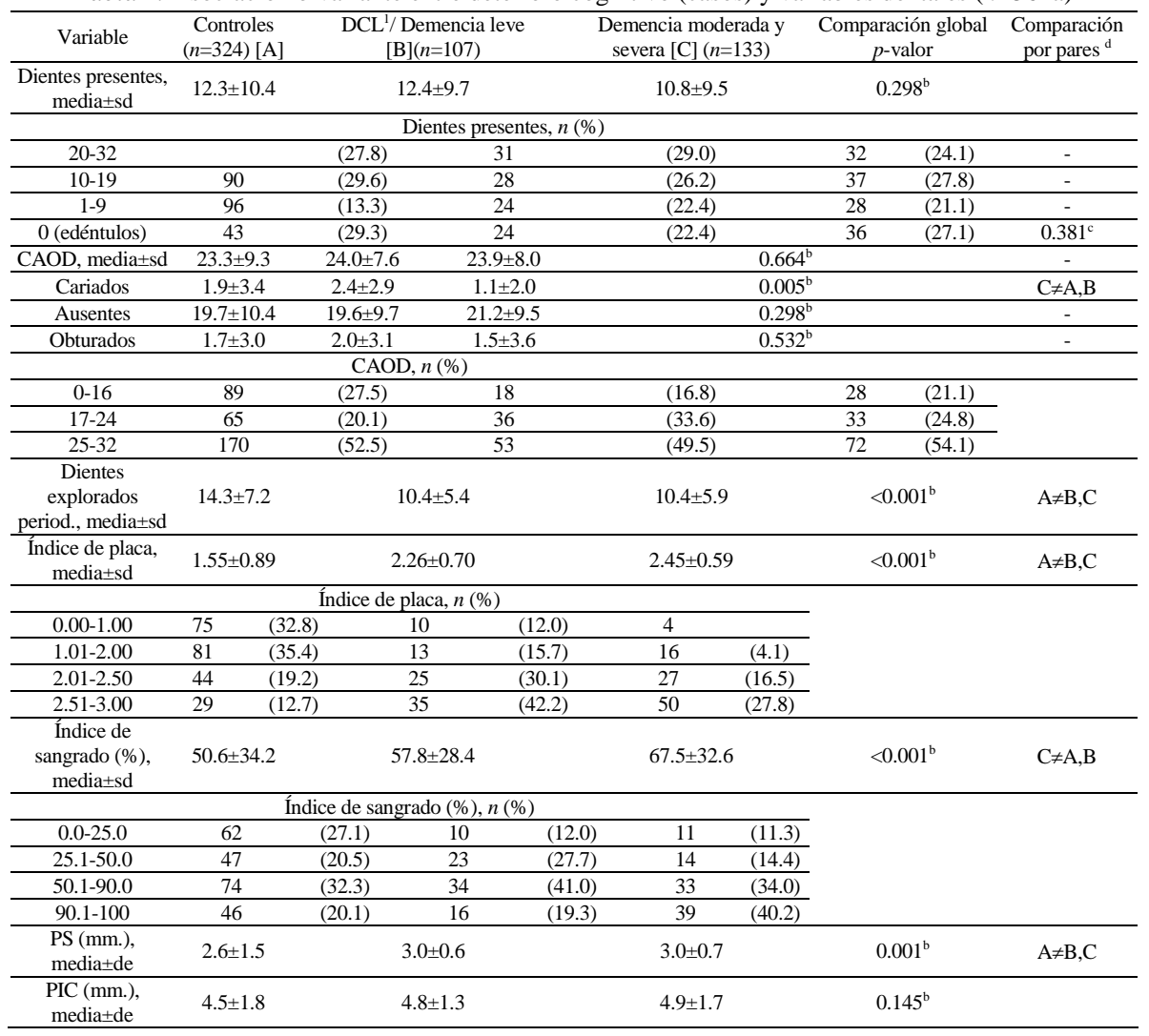


Tabla 2 (continuación). Asociación bivariante entre deterioro cognitivo (casos) y variables dentales

\begin{tabular}{|c|c|c|c|c|c|c|c|}
\hline \multicolumn{8}{|c|}{$(n=564 a)$} \\
\hline $\begin{array}{c}\mathrm{PIC}(\%>3 \mathrm{~mm} . \\
\text { media } \pm \text { sd }\end{array}$ & $65.3 \pm 35.2$ & & \multicolumn{2}{|c|}{$76.2 \pm 25.6$} & $73.9 \pm 31.4$ & $0.011^{\mathrm{b}}$ & $\mathrm{A} \neq \mathrm{B}, \mathrm{C}$ \\
\hline \multicolumn{6}{|c|}{ PIC $(\%>3 \mathrm{~mm}),. n(\%)$} & & \\
\hline $\begin{array}{c}0.0-32.9 \\
\text { (ausente/leve) }\end{array}$ & 56 & $(24.5)$ & 5 & (6.0) & 13 & & \\
\hline $\begin{array}{c}33.0-66.9 \\
\text { (moderado) }\end{array}$ & 47 & $(20.5)$ & 22 & (26.5) & 17 & & \\
\hline $67.0-100$ (severo) & 126 & $(55.0)$ & 56 & $(67.5)$ & 67 & & \\
\hline
\end{tabular}

Para el análisis periodontal, se exploraron una media de 14.3 dientes en los controles y de 10.4 en los casos. Tanto el índice de placa como el índice de sangrado muestran diferencias claramente significativas entre los casos y los controles, donde se observa un aumento progresivo, de manera que en el grupo control se observa un índice de placa de 1.55 , siendo éste de 2.26 y 2.45 para los grupos de deterioro cognitivo leve y demencia moderada-severa respectivamente. Lo mismo se observa en el índice de sangrado, donde existe un 50.6\% en el grupo control y aumenta hasta un $57.8 \%$ y un $67.5 \%$ en los casos mencionados anteriormente.

En cuanto a la profundidad de sondaje no existen diferencias entre ambos grupos casos, pero si hay una diferencia significativa con el grupo control, siendo de 3 $\mathrm{mm}$ para los casos y $2.6 \mathrm{~mm}$ para los controles.

La gravedad de enfermedad periodontal medida con la pérdida de inserción clínica fue mayoritariamente severa en todos los grupos, siendo del 55\% en los controles y del $68.3 \%$ en los casos. Estos resultados concuerdan con otros estudios en los que se establece que la prevalencia de inflamación gingival y la placa es mayor en pacientes institucionalizados con demencia y discapacidad que los que no presentan deterioro, a pesar de que reciben mayor asistencia y cuidados dentales (Chen, Clark, y Naorungroj, 2013; Philip, Rogers, Kruger, y Tennant, 2011).

El análisis multivariante (Tabla 3) refleja claramente como la enfermedad periodontal clínica se asocia con el grado de deterioro cognitivo/demencia, una vez ajustado por variables clásicas que pueden afectar a dicha asociación como son: edad, sexo, nivel de estudios, tabaco o alcohol. No ocurre lo mismo con otras variables odontológicas como el número de dientes presentes o los índices de caries. Destaca el índice de sangrado, que muestra una diferencia significativa y es 16.81 veces mayor en el grupo de demencia moderada-severa que en el grupo control. También muestran diferencias significativas el índice de placa, la profundidad de sondaje y la pérdida de inserción clínica. 
Tabla 3. Medias y desviaciones típicas de la interacción de las variables evaluadas

\begin{tabular}{|c|c|c|c|c|c|c|c|c|c|}
\hline \multirow[b]{2}{*}{ Variable } & \multirow[b]{2}{*}{$\mathrm{n}^{\mathrm{a}}$ total } & \multirow[b]{2}{*}{$\begin{array}{c}\mathrm{n}^{\mathrm{b}} \\
\text { dentado }\end{array}$} & \multicolumn{7}{|c|}{ Modelo (variable dependiente) } \\
\hline & & & $\begin{array}{c}\text { Dientes } \\
\text { presente } \beta \pm \text { se }\end{array}$ & $\begin{array}{c}\mathrm{CAOD} \\
\beta \pm \mathrm{se}\end{array}$ & $\begin{array}{l}\text { Índice de } \\
\text { placa } \beta \pm \text { se }\end{array}$ & $\begin{array}{c}\text { Índice de } \\
\text { sangrado } \beta \pm \text { se }\end{array}$ & $\begin{array}{c}\text { Profundidad de } \\
\text { sondaje } \beta \pm \text { se }\end{array}$ & $\begin{array}{c}\text { PIC (mm.) } \\
\beta \pm \text { se }\end{array}$ & $\begin{array}{l}\mathrm{PIC}(\%>3 \\
\text { mm. } \beta \pm \text { se }\end{array}$ \\
\hline Grupo & & & $p=0.116$ & $p=0.080$ & $p<0.001$ & $p<0.001$ & $p<0.001$ & $p=0.050$ & $p=0.004$ \\
\hline $\begin{array}{c}\text { Demencia } \\
\mathrm{mod} / \mathrm{sev}\end{array}$ & 133 & 97 & $-1.65 \pm 0.91$ & $0.63 \pm 0.80$ & $0.91 \pm 0.08$ & $16.81 \pm 3.98$ & $0.44 \pm 0.13$ & $0.46 \pm 0.22$ & $8.97 \pm 3.99$ \\
\hline $\begin{array}{c}\mathrm{DCL}^{1} / \text { Demencia } \\
\text { leve }\end{array}$ & 107 & 83 & $-1.66 \pm 1.11$ & $2.09 \pm 0.93$ & $0.74 \pm 0.10$ & $7.45 \pm 3.91$ & $0.48 \pm 0.12$ & $0.36 \pm 0.19$ & $12.05 \pm 3.80$ \\
\hline Control & 324 & 229 & 0.00 & 0.00 & 0.00 & 0.00 & 0.00 & 0.00 & 0.00 \\
\hline Edad & & & $p<0.001$ & $p<0.001$ & $p=0.003$ & $p=0.319$ & $p=0.003$ & $p<0.001$ & $p=0.013$ \\
\hline Años & 564 & 409 & $-0.44 \pm 0.05$ & $0.38 \pm 0.05$ & $0.01 \pm 0.00$ & $-0.23 \pm 0.23$ & $0.02 \pm 0.01$ & $0.04 \pm 0.01$ & $0.56 \pm 0.23$ \\
\hline Sexo & & & $p=0.493$ & $p=0.031$ & $p=0.312$ & $p=0.046$ & $p=0.413$ & $p=0.293$ & $p=0.568$ \\
\hline Hombre & 217 & 160 & $0.60 \pm 0.87$ & $-1.64 \pm 0.76$ & $0.09 \pm 0.08$ & $7.31 \pm 3.66$ & $-0.11 \pm 0.13$ & $0.20 \pm 0.19$ & $2.05 \pm 3.59$ \\
\hline Mujer & 347 & 249 & 0.00 & 0.00 & 0.00 & 0.00 & 0.00 & 0.00 & 0.00 \\
\hline Studios & & & $p=0.092$ & $p=0.014$ & $p=0.006$ & $p=0.125$ & $p=0.002$ & $p=0.442$ & $p=0.449$ \\
\hline Univ/Secundaria & 58 & 42 & $2.01 \pm 1.43$ & $0.44 \pm 1.18$ & $-0.14 \pm 0.12$ & $-10.38 \pm 6.10$ & $0.20 \pm 0.25$ & $0.23 \pm 0.35$ & $-0.16 \pm 6.16$ \\
\hline Primaria & 113 & 86 & $2.94 \pm 1.25$ & $-2.39 \pm 1.08$ & $-0.35 \pm 0.11$ & $-10.11 \pm 4.67$ & $-0.49 \pm 0.19$ & $-0.19 \pm 0.30$ & $-5.71 \pm 5.24$ \\
\hline $\begin{array}{c}\text { Primaria } \\
\text { incompleta }^{\mathrm{a}}\end{array}$ & 237 & 191 & $2.19 \pm 1.03$ & $-2.09 \pm 0.85$ & $-0.25 \pm 0.09$ & $-5.37 \pm 4.02$ & $-0.41 \pm 0.16$ & $-0.21 \pm 0.22$ & $1.08 \pm 4.33$ \\
\hline Ninguno & 156 & 90 & 0.00 & 0.00 & 0.00 & 0.00 & 0.00 & 0.00 & 0.00 \\
\hline Tabaco & & & $p=0.163$ & $p=0.689$ & $p=0.593$ & $p=0.910$ & $p=0.624$ & $p=0.505$ & $p=0.855$ \\
\hline Sí & 44 & 32 & $-2.49 \pm 1.78$ & $0.65 \pm 1.63$ & $0.09 \pm 0.17$ & $0.82 \pm 7.28$ & $-0.11 \pm 0.22$ & $0.21 \pm 0.32$ & $1.14 \pm 6.22$ \\
\hline $\mathrm{No}^{\mathrm{b}}$ & 520 & 377 & 0.00 & 0.00 & 0.00 & 0.00 & 0.00 & 0.00 & 0.00 \\
\hline Alcohol, $n(\%)$ & & & $p=0.015$ & $p<0.001$ & $p=0.001$ & $p=0.323$ & $p=0.353$ & $p=0.146$ & $p=0.042$ \\
\hline$\geq 1$ vez/día & 25 & & $-5.08 \pm 1.88$ & $7.57 \pm 1.32$ & $0.60 \pm 0.17$ & $-8.99 \pm 8.32$ & $0.39 \pm 0.27$ & $0.74 \pm 0.43$ & $14.61 \pm 6.87$ \\
\hline Eventualmente & 65 & 20 & $-2.32 \pm 1.40$ & $2.37 \pm 1.19$ & $0.12 \pm 0.12$ & $-7.03 \pm 5.73$ & $0.08 \pm 0.21$ & $0.40 \pm 0.33$ & $9.06 \pm 5.05$ \\
\hline $\mathrm{No}^{\mathrm{c}}$ & 474 & 43 & 0.00 & 0.00 & 0.00 & 0.00 & 0.00 & 0.00 & 0.00 \\
\hline
\end{tabular}

Algunos parámetros de enfermedad periodontal como el índice de placa, índice gingival y porcentaje de sangrado, profundidad de sondaje y pérdida de inserción clínica han sido previamente evaluados en pacientes diagnosticados de Alzheimer que presentaban distinto grado de deterioro cognitivo, dando como resultado que todos los parámetros evaluados fueron mayores en los pacientes con Alzheimer, y que el estado periodontal empeoraba con la progresión de la enfermedad (Martande, 2014), tal y como se demuestra en nuestro estudio. Otros artículos han mostrado una tendencia similar, haciendo referencia en estos casos a que una peor función cognitiva se asocia con un mayor número de dientes cariados, dientes ausentes y un peor estado periodontal en personas mayores (Wu, Plassman, Crout, y Liang, 2008; Yu y Kuo, 2008).

\section{DISCUSIÓN Y CONCLUSIONES}

Tal y como hemos observado en este estudio, el progreso del deterioro cognitivo que presentan los pacientes de mayor edad se asocia con un empeoramiento global de su estado de salud oral y con una incapacidad progresiva de realizar una adecuada higiene bucodental, así como recibir asistencia odontológica. Los individuos con mayor deterioro cognitivo presentan una mayor extensión y gravedad de enfermedad periodontal y ésta aumenta conforme se produce el avance de la enfermedad 
neurodegenerativa. Este es un hecho fundamental a tener en cuenta tanto por los odontólogos como por el personal que se encarga del cuidado de estos pacientes. Los planes de tratamiento odontológicos deben ser individualizados por cada paciente y centro, y ajustados a su nivel cognitivo y capacidad funcional, pudiendo ser éste modificado a medida que avanza su demencia, y por tanto las expectativas y prioridades del paciente. Así mismo, sería recomendable establecer un adecuado plan de prevención y control de placa bacteriana, enseñando técnicas de higiene y educación sanitaria mientras el paciente mayor sea capaz. También es de vital importancia, instruir a los cuidadores y familiares en el adecuado mantenimiento oral de estos pacientes y sus necesidades.

El impacto del deterioro cognitivo/demencia sobre la salud oral se refleja básicamente en el mal estado de salud periodontal, valorada ésta mediante el índice de placa, índice de sangrado, profundidad de bolsa y pérdida de inserción epitelial.

\section{REFERENCIAS}

Beck, J.D., y Löe, H. (1993). Epidemiological principles in studying periodontal diseases. Periodontology, 2(1), 34-45.

Bermejo-Pareja, F., Benito-León, J., Vega, S., Medrano, M., y Román, G. (2008). Incidence and subtypes of dementia in three elderly populations of central Spain. Journal of the Neurological Sciences, 264(1-2), 63-72.

Brennan, L.J., y Strauss, J. (2014). Cognitive Impairment in Older Adults and Oral Health Considerations. Dental Clinics of North America, 58(4), 815-828

Carnero-Pardo, C., y Montoro-Ríos, M.T. (2004). Test de las Fotos. Revista Neurológica, 39, 80106.

Chen, X., Clark, J.J., y Naorungroj, S. (2013). Oral health in nursing home residents with different cognitive statuses. Gerodontology, 30(1), 49-60.

Gil-Montoya, J.A., Sanchez-Lara, I., Carnero-Pardo, C., Fornieles, F., Montes, J., Vílchez, R., y Bravo, M. (2015). Is Periodontitis a Risk Factor for Cognitive Impairment and Dementia? A Case-Control Study. Journal of Periodontology, 86(2), 244-253.

Kamer, A.R., Dasanayake, A.P., Craig, R.G., Glodzik-Sobanska, L., Bry, M., y Leon, M.J. (2008). Alzheimers Disease and Peripheral Infections: The Possible Contribution from Periodontal Infections, Model and Hypothesis. Journal of Alzheimers Disease, 13(4), 437-449.

Lee, K.H., Wu, B., y Plassman, B.L. (2013). Cognitive Function and Oral Health-Related Quality of Life in Older Adults. Journal of the American Geriatrics Society, 61(9), 1602-1607.

Lexomboon, D., Trulsson, M., Wårdh, I., y Parker, M.G. (2012). Chewing Ability and Tooth Loss: Association with Cognitive Impairment in an Elderly Population Study. Journal of the American Geriatrics Society, 60(10), 1951-1956.

Martande, S.S., Pradeep, A.R., Singh, S.P., Kumari, M., Suke, D.K., Raju, A.P., y Chatterji, A. (2014). Periodontal Health Condition in Patients With Alzheimer's Disease. American Journal of Alzheimers Disease \& Other Dementiasr, 29(6).

Naorungroj, S., Slade, G., Beck, J., Mosley, T., Gottesman, R., Alonso, A., y Heiss, G. (2013). Cognitive Decline and Oral Health in Middle-aged Adults in the ARIC Study. Journal of Dental Research, 92(9), 795-801. 
Nilsson, H., Berglund, J., y Renvert, S. (2014). Tooth loss and cognitive functions among older adults. Acta Odontologica Scandinavica, 72(8), 639-644.

Noble, J.M., Scarmeas, N., y Papapanou, P.N. (2013). Poor Oral Health as a Chronic, Potentially Modifiable Dementia Risk Factor: Review of the Literature. Current Neurology and Neuroscience Reports, 13(10).

Pedro-Cuesta, J.D., Virués-Ortega, J., Vega, S., Seijo-Martínez, M., Saz, P., Rodríguez, F., y Barrio, J.L. (2009). Prevalence of dementia and major dementia subtypes in Spanish populations: A reanalysis of dementia prevalence surveys, 19902008. BMC Neurology, 9(1).

Petersen, R.C. (2011). Mild Cognitive Impairment. New England Journal of Medicine, 364(23), 2227-2234

Philip, P., Rogers, C., Kruger, E., y Tennant, M. (2011). Oral hygiene care status of elderly with dementia and in residential aged care facilities. Gerodontology, 29(2).

Singhrao, S.K., Harding, A., Simmons, T., Robinson, S., Kesavalu, L., y Crean, S. (2014). Oral Inflammation, Tooth Loss, Risk Factors, and Association with Progression of Alzheimers Disease. Journal of Alzheimers Disease, 42(3), 723-737.

Wu, B., Plassman, B.L., Crout, R.J., y Liang, J. (2008). Cognitive Function and Oral Health Among Community-Dwelling Older Adults. The Journals of Gerontology Series A: Biological Sciences and Medical Sciences, 63(5), 495-500.

Yu, Y., y Kuo, H. (2008). Association Between Cognitive Function and Periodontal Disease in Older Adults. Journal of the American Geriatrics Society, 56(9), 1693-1697.

Recibido: 6 de noviembre de 2018

Recepción Modificaciones: 19 de noviembre de 2018

Aceptado: 21 de noviembre de 2018 